\title{
Double-Tapered-Waveguide Distributed Feedback Lasers for High-Power Single-Mode Operation
}

\author{
S. F. Yu
}

\begin{abstract}
Theoretical analysis of double-tapered-waveguide distributed feedback semiconductor laser for stable single-mode and high-power operation is presented. It is found that the single-mode behavior of a distributed feedback laser with large coupling-length product can be improved significantly by the double-tapered-waveguide structure.
\end{abstract}

Index Terms - Distributed feedback lasers, double-tapered waveguide, modeling, semiconductor lasers.

\section{INTRODUCTION}

$\mathbf{H}$ IGH-POWER $\lambda / 4$ phase-shifted distributed feedback (DFB) lasers with stable single-mode operation are desirable for wavelength-selective applications such as frequency doubling, atomic spectroscopy, as well as for pumping of $\mathrm{Er}^{3+}$-doped fiber amplifier. However, the side-mode suppression ratio (SMSR) can be degraded due to the longitudinal spatial hole burning (LSHB) especially for devices with large coupling-length product $(\kappa L>1.25)$ operating at high output power [1]. This is because the gain margin of the DFB laser is deteriorated by the nonuniform distribution of refractive index arisen from the LSHB. However, a high $\kappa L$ device is far more attractive due to low threshold current density, relatively large AM bandwidth, small linewidth-power product, as well as low reflection sensitivity [2].

Various methods have been proposed in order to overcome the degradation of SMSR at high output power and some laser structures have demonstrated stable single-mode operation [3]-[5]. For example, a linearly chirped grating structure to suppress nonuniform field distribution has been reported [4]. However, direct implementation of chirped grating DFB laser is difficult such that an alternative approach using a bent waveguide is proposed to realize chirped grating DFB lasers [5]. On the other hand, a DFB laser with a phase-adjustment region (PAR) is another way to obtain single-mode devices with less pronounced maximum in the intensity distribution than that of a discrete $\lambda / 4$ phase-shifted DFB laser [1], [3].

Maximum output power of semiconductor lasers is also limited by the catastrophic optical damage (COD). COD is due to local melting caused by heating as a result of intense nonradiative electron-hole recombination at the device's facets. The local melting may be initiated at defects or at defectfree cleaved facets [6], [7]. Therefore, a wide-stripe width is desired in order to reduce the power density at facets. In

\footnotetext{
Manuscript received March 11, 1996; revised September 16, 1996.

The author is with the Department of Electrical and Electronic Engineering, University of Hong Kong, Hong Kong.

Publisher Item Identifier S 0018-9197(97)00355-2.
}

the design of high-power lasers, ridge waveguide structure is usually employed such that single-lateral-mode operation can be maintained at wide stripe width $(\geq 5 \mu \mathrm{m})$ due to weak lateral guiding effects [8], [9]. However, the disadvantage of adopting a ridge waveguide structure is high-threshold current density due to spreading of optical intensity outside the active stripe region. Alternative, high-power lasers with buried heterostructure $(\mathrm{BH})$ can be utilized at the expense of single-lateral-mode operation [10].

In the design of high-power single-mode DFB lasers, it is required: 1) to reduce the influence of LSHB for stable singlelongitudinal-mode operation; 2) to maintain stable singlelateral-mode operation; 3) to minimize power density at facets; and 4) to reduce threshold current density. Recently, a doubletapered-waveguide (DTWG) DFB laser with BH was proposed [11] to achieve the above requirements for effective highpower single-mode operation. In this paper, we present a detailed analysis on the high power behavior of DTWG-DFB lasers. It can be shown that stable single-mode and high-power operation can be achieved even in large $\kappa L$ devices for a wide range of waveguide dimension (i.e., length and width of the tapered and phase-adjustment region).

This paper is organized as follows. In Section II, a selfconsistent model including the longitudinal variation of carrier concentration, photon density, and refractive index is utilized to study the steady-state characteristics of DFB lasers with a double-tapered waveguide. The spatial filtering effects of the tapered waveguide sections are also taken into account by using the effective index method [12]. In Section III, the influence of double-tapered geometry on the high-power behavior of DFB lasers is analyzed. The efficiency of using wide-stripe width to reduce the facets' power density is discussed. A comparison between double-tapered waveguide and discrete $\lambda / 4$ phase-shifted DFB lasers is also given. Furthermore, the possibility of using high-reflection (HR) coating in DTWG-DFB lasers for single-mode high-power operation is investigated. Finally, a brief conclusion is given in Section IV.

\section{Operation PRINCIPLE AND MODEL}

\section{A. Operation Principle of Double-Tapered-Waveguide Distributed Feedback Lasers}

Fig. 1 shows the DTWG-DFB lasers with BH. The laser consists of two tapered waveguides which are utilized to suppress higher order lateral modes by the diffraction effects through the center narrow waveguide. Power density at facets 


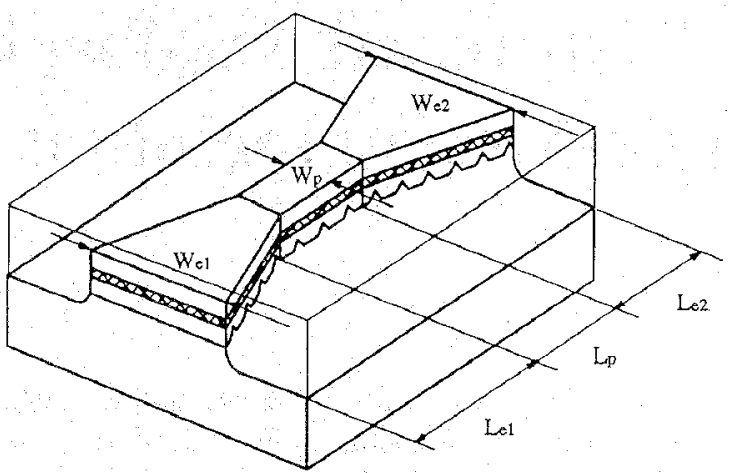

Fig. 1. Schematic of a DFB laser with double-tapered-waveguide structure.

is reduced by the spreading of optical field such that the COD level is improved. The center waveguide region can be considered as a PAR which introduces a $\lambda / 4$ phase-shifted to the propagation fields for single-longitudinal-mode oscillation. Therefore, a DFB laser with DTWG can provide stable singlelateral- and longitudinal-mode oscillation. Furthermore, the LSHB of carrier concentration is also affected by the DTWG. This is because narrow PAR reduces peak intensity of the propagation fields and severe LSHB can be avoided. Therefore, single-mode emission can be maintained in devices with large $\kappa L$ operating at a high power level. In addition, devices with $\mathrm{BH}$ exhibit lower threshold current density than ridge waveguide [8], [9] due to strong lateral optical and electrical confinement. It is also expected that the carrier confinement near the facets can have significant improvement in the COD level as it is also a current-induced phenomenon of lasers [13]. Another advantage of the proposed device is that it is a simple processing technique and compatible with existing fabrication technologies of the conventional semiconductor lasers.

\section{B. Laser Model}

The propagation of forward and reverse fields, $F$ and $R$, along the laser cavity with tapered waveguide can be described by the modified coupled-wave equations as follows:

$$
\pm \frac{\partial}{\partial z}\left[\begin{array}{l}
F \\
R
\end{array}\right]=\left[\frac{1}{2} \Gamma_{y}\left(\Gamma_{x} G-\alpha_{s}\right)+i \delta \beta\right]\left[\begin{array}{l}
F \\
R
\end{array}\right]+i \kappa\left[\begin{array}{l}
F \\
R
\end{array}\right]
$$

where $\alpha_{s}$ is the total absorption and scattering loss of the QW waveguide, $\kappa$ is the coupling coefficient, and $\Gamma_{y}$ and $\Gamma_{x}$ are the optical confinement factors in the lateral and transverse directions, respectively.

It must be noted that the round-trip conditions of DTWGDFB lasers are affected by the waveguide dimension. This is because the longitudinal propagation constant is changed according to the stripe width of waveguide, such that the deviation in propagation constant from Bragg constant is also dependent on the waveguide dimension. Therefore, the magnitude of optical confinement factor and coupling coefficient are varied along the laser cavity. The dependence of $\Gamma_{y}, \kappa$, and $\delta \beta$ on the stripe width of the waveguide can be evaluated by the effective-index method [12] (see Appendix $A$ for a detailed derivation).
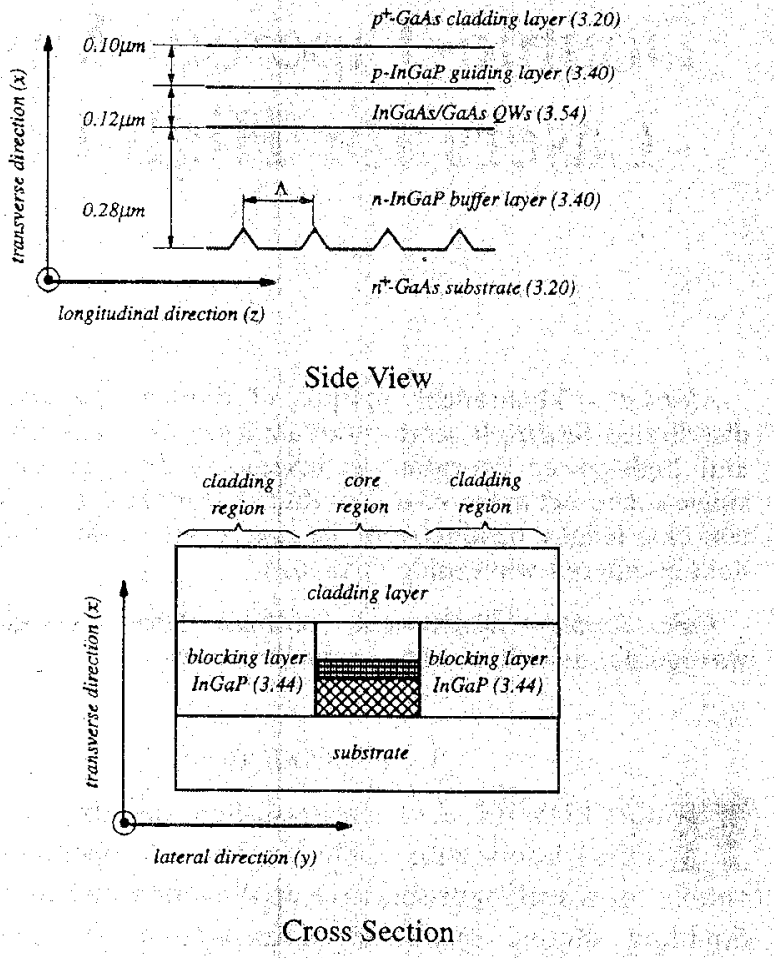

Fig. 2. Schematic of the side view and cross section of the BHDFB laser investigated in this paper.

The modal gain, $G$, can be approximated by

$$
G=a_{N}\left(N-N_{0}\right)
$$

where $a_{N}$ is the differential gain, $N$ is the carrier concentration and $N_{o}$ is the carrier concentration at transparency. The deviation from Bragg's condition, $\delta \beta$, is given as

$$
\delta \beta(z)=\frac{2 \pi}{\lambda_{0}}\left(n_{\text {eff }}+\Delta n_{r} \Gamma_{c}\right)-\frac{\pi}{\Lambda}
$$

where $\lambda_{0}$ is the operating wavelength, $\Lambda$ is the period of grating, $n_{\text {eff }}$ is the effective refractive index of the waveguide; and $\Delta n_{r}$ is the carrier-induced index change given by

$$
\Delta n_{r}=\frac{\alpha_{m} \lambda_{0}}{4 \pi} a_{N} \Delta N
$$

where $\alpha_{m}$ is the linewidth enhancement factor and $\Delta N$ is the variation of carrier concentration above threshold. For threshold analysis, we assume that the carrier concentration is uniform along the waveguide region. The threshold gain and lasing frequency of DTWG-DFB lasers can be obtained by solving (1) with the appropriate boundary conditions at the facets. In addition, the nonuniform distribution of $\kappa, \delta \beta$, and $\Gamma_{y}$ can be taken into account by the transfer matrix method [14] as shown in Appendix B. The device is split into a cascaded section and each section is represented by a transfer matrix. It is assumed that in each section $\kappa, \delta \beta$, and $\Gamma_{y}$ are constant but these parameters can vary in different sections. The output power $P_{h \nu}$ from the left facet is given by

$$
P_{h \nu}=\frac{1}{2} v_{g} h \nu\left(1-\left|r_{L}\right|^{2}\right) A_{\mathrm{eff}} P(0)
$$

where $v_{g}$ is the group velocity, $h$ is the Plank's constant, $\nu$ is the oscillation frequency, $r_{L}$ is the left facet reflectivity 


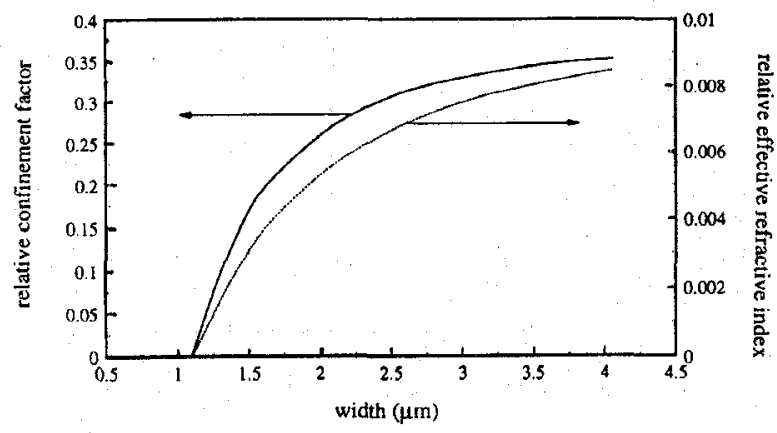

Fig. 3. The variation of relative confinement factor, $\Delta \Gamma_{y}$, and relative effective refractive index, $\Delta n_{\text {eff }}$, with the stripe width.

TABLE I

\begin{tabular}{l|l}
\hline Parameters (symbol) & Magnitude \\
\hline \hline Carrier lifetime $(\tau)$ & $3 \times 10^{-9} \mathrm{~s}$ \\
\hline Differential gain $\left(\mathrm{a}_{\mathrm{N}}\right)$ & $3 \times 10^{-16} \mathrm{~cm}^{2}$ \\
\hline Transparency carrier density $\left(\mathrm{N}_{\mathrm{o}}\right)$ & $1.5 \times 10^{18} \mathrm{~cm}^{-3}$ \\
\hline Linewidth enhancement factor $\left(\alpha_{\mathrm{m}}\right)$ & 5.0 \\
\hline Absorption and scattering loss $\left(\alpha_{\mathrm{S}}\right)$ & $40 \mathrm{~cm}^{-1}$ \\
\hline Effective group refractive index $\left(\mathrm{n}_{\mathrm{g}}\right)$ & 3.70 \\
\hline Thickness of the active layer $(\mathrm{d})$ & $0.2 \mu \mathrm{m}$ \\
\hline Approximate emission wavelength $\left(\lambda_{\mathrm{O}}\right)$ & $0.98 \mu \mathrm{m}$ \\
\hline Transverse optical confinement factor $\left(\Gamma_{\mathrm{X}}\right)$ & 0.35 \\
\hline Period of grating $(\Lambda)$ & $0.145 \mu \mathrm{m}$ \\
\hline Group velocity $\left(v_{\mathrm{g}}\right)$ & $8 \times 10^{9} \mathrm{~cm} / \mathrm{s}$ \\
\hline magnitude of Left facet reflectivity $\left(\mathrm{r}_{\mathrm{L}} \mathrm{l}\right)$ & 0 \\
\hline magnitude of Right facet reflectivity $\left(\mathrm{r}_{\mathrm{R}}\right)$ & $0,0.55$ \\
\hline
\end{tabular}

and $P\left(=|F|^{2}+|R|^{2}\right)$ is the photon density at $z=0 . A_{\text {eff }}$ is the effective area of the output beam and is approximated by multiplying the full-width half-maximum (FWHM) optical intensity distribution of the fundamental transverse and lateral modes.

For the above threshold analysis, the longitudinal distribution of carrier concentration can also be described by the transfer matrix. In this case, the carrier concentration, photon density, and refractive index are also allowed to vary between sections [15]. In addition, the influence of external current injection on the longitudinal distribution of carrier concentration is included in the analysis by the carrier rate equation

$$
\frac{d N}{d t}=\frac{J}{e d}-\frac{N}{\tau}-v_{g} a_{N}\left(N-N_{o}\right) P
$$

where $d$ is the total thickness of active layer, $J$ is the injection current density, $e$ is the electron charge, and $\tau$ is the carrier lifetime. Now, the above threshold behavior of devices can be determined by solving (1) and (6) in a self-consistent manner at a particular injection current.

\section{Simulation Results}

\section{A. Material Composition of Buried Heterostructure Waveguide}

The laser structure used in our analysis is the BH InGaAs-GaAs $(0.98 \mu \mathrm{m})$ quantum-well DFB laser with firstorder grating. Material composition and refractive index profile of the device are shown in Fig. 2. The laser is composed of
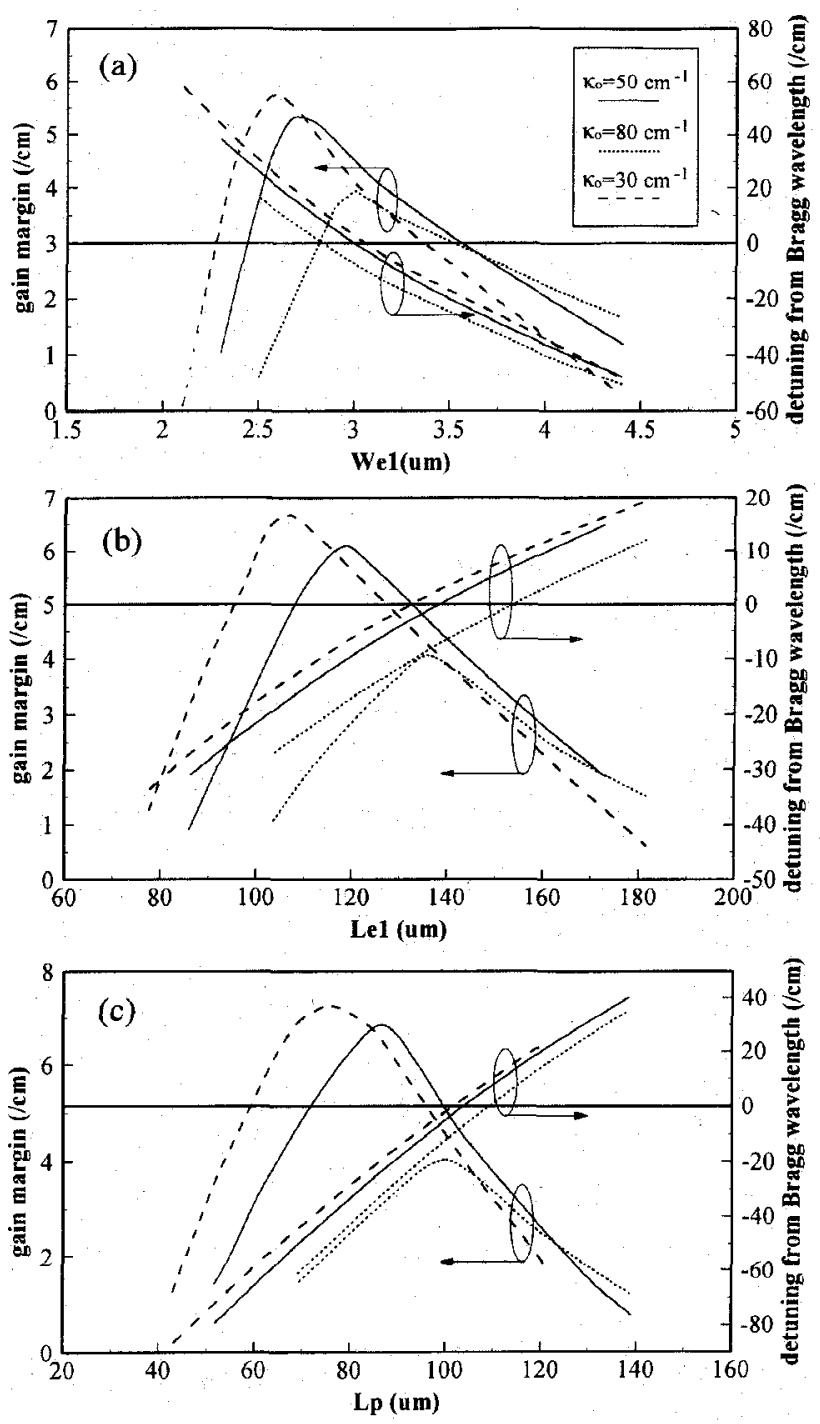

Fig. 4. The variation of gain margin and dominant mode detuning from Bragg wavelength with (a) $W_{e 1}$, (b) $L_{e 1}$, and (c) $L_{p}$ for devices with $\kappa_{0}=$ $50 \mathrm{~cm}^{-1}$ (solid line), $\kappa_{0}=80 \mathrm{~cm}^{-1}$ (dotted line), and $\kappa_{0}=30 \mathrm{~cm}^{-1}$ (dashed line).

five layers of semiconductor materials: the $p^{+}-\mathrm{GaAs}$ cladding layer, $p$-InGaP guiding layer, the InGaAs-GaAs QW's active region, the $n$-InGaP buffer layer and $n^{+}$-GaAs substrate. The current blocking layers are of InGaP material which provide optical and electrical confinement along the lateral direction. Using the refractive-index distribution shown in Fig. $2, \Gamma_{x}$ is found to be 0.35 . The effective refractive index $\left(n_{\text {eff }}\right)$ in the core and cladding region are calculated for the fundamental lateral mode and is equal to 3.383 and 3.380 , respectively. Other device parameters used in the model can be found in Table I.

\section{B. Steady-State Characteristics of DTWG DFB Lasers}

To simplify our calculation, the relative (to the PAR) effective refractive index $\Delta n_{\mathrm{eff}}$ and transverse confinement factor $\Delta \Gamma_{y}$ of the tapered waveguide are considered in the analysis. Fig. 3 plots the variation of $\Delta n_{\text {eff }}$ and $\Delta \Gamma_{y}$ with stripe width. It is assumed that the width of PAR $W_{p}$ is $1.0 \mu \mathrm{m}$. The 

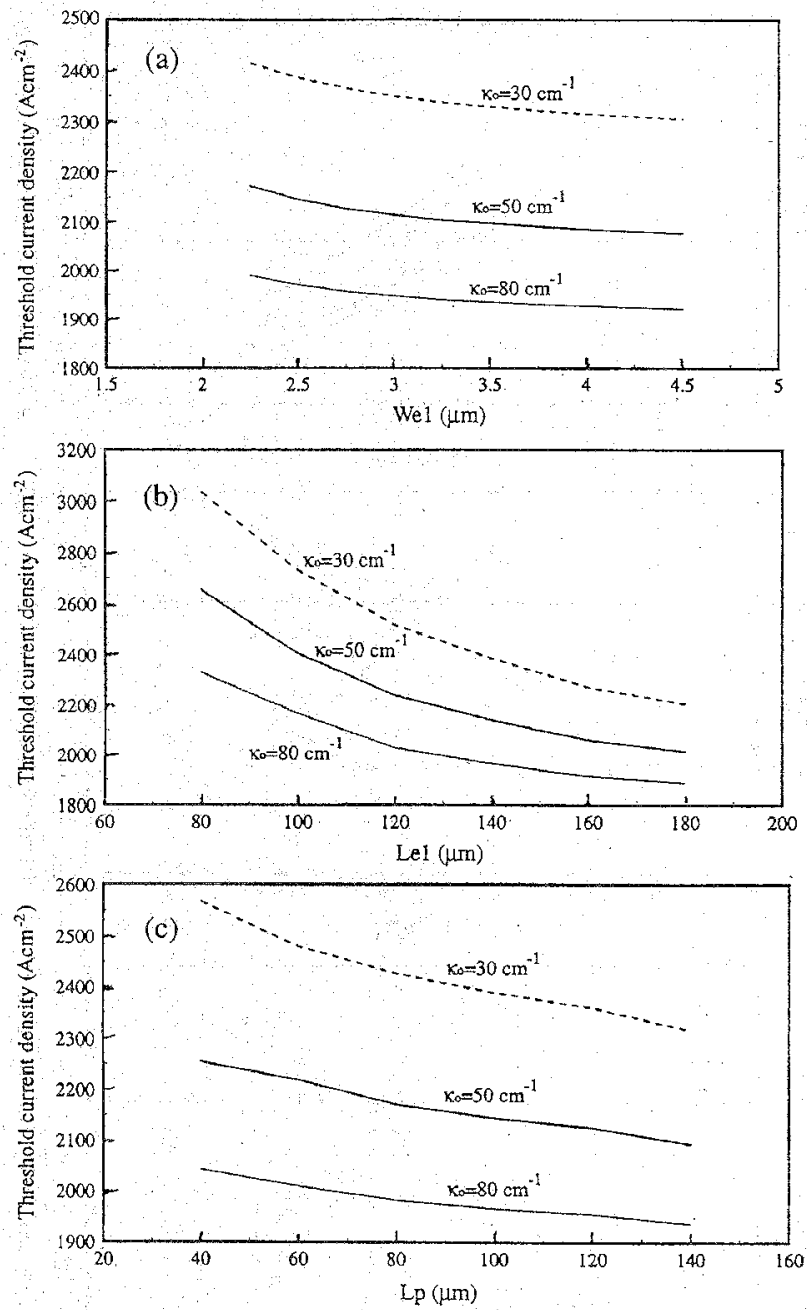

Fig. 5. The variation of threshold current density with (a) $W_{e 1}$, (b) $L_{e 1}$, and (c) $L_{p}$ for devices with $\kappa_{0}=50 \mathrm{~cm}^{-1}$ (solid line), $\kappa_{0}=80 \mathrm{~cm}^{-1}$ (dotted line), and $\kappa_{0}=30 \mathrm{~cm}^{-1}$ (dashed line).

relative coupling coefficient $\triangle \kappa$ can also be determined from $\Delta \Gamma_{y}$ [see (A6)]. $\Gamma_{y}$ and $n_{\text {eff }}$ of waveguide with width $1 \mu \mathrm{m}$ has a magnitude equal to 0.6545 and 3.2828 , respectively. In addition, the modal gain, $\bar{G}$, of the oscillation modes is defined as

$$
\bar{G}=\frac{1}{L} \int_{0}^{L} \Gamma_{y}(z) a_{N}\left(N(z)-N_{o}\right) d z
$$

where $N(z)$ is the longitudinal distribution of carrier concentration.

Fig. 4 shows the gain margin and oscillation wavelength of the dominant mode yersus (a) width of tapered waveguide $W_{e 1}$ (with $L_{e 1}=140 \mu \mathrm{m}$ and $L_{p}=100 \mu \mathrm{m}$ ), (b) length of tapered waveguide $L_{e 1}$ (with $W_{e 1}=3 \mu \mathrm{m}$ and $I_{p}=100 \mu \mathrm{m}$ ), and (c) length of PAR $L_{p}$ (with $W_{e 1}=3 \mu \mathrm{m}$ and $L_{e 1}=140 \mu \mathrm{m}$ ) at threshold. In the calculations, $\kappa_{0}$ (coupling coefficient at the PAR) is varied between $30 \mathrm{~cm}^{-1}$ and $80 \mathrm{~cm}^{-1}$. The width $W_{e 2}$ and length $L_{e 2}$ of the second tapered section is assumed to be varied with $W_{e 1}$ and $L_{e 1}$, respectively. The results of Fig. 4 can be summarized as follows.

1) The maximum gain margin is detuned from the Bragg wavelength:
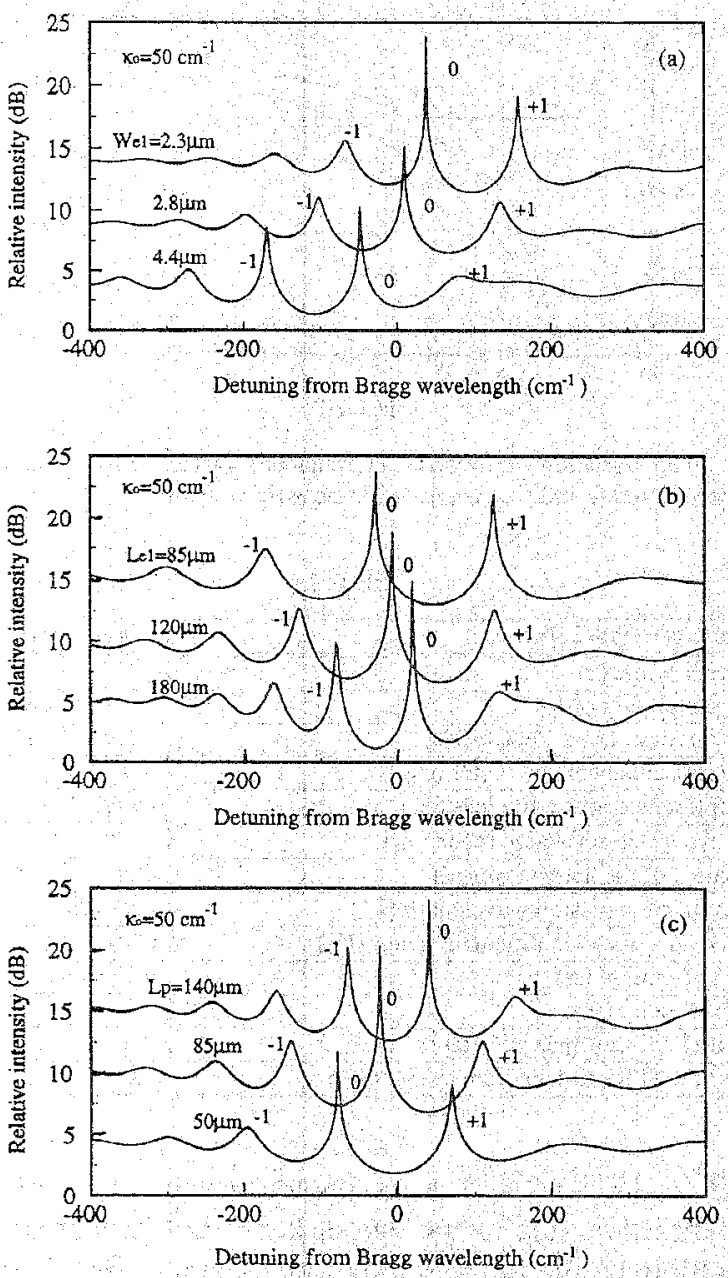

Fig. 6. The spectrum profile of DTWG with $\kappa_{0}=50 \mathrm{~cm}-1$ for different dimensions of $W_{e 1}, L_{e 1}$, and $L_{p}$.

2) The gain margin peak is affected by the waveguide dimension.

3) The increase of $W_{e 1}\left(L_{e 1}\right.$ and $\left.L_{p}\right)$ introduces a negative (positive) detuning on the dominant mode.

The results show that the waveguide dimension of the DTWG structure has significant influence on the threshold gain and round-trip conditions. However, the discrete $\lambda / 4$ (or PAR) DFB lasers exhibit different characteristics. 1 ) the maximum gain margin appears at the Bragg wavelength; and 2) the lasing. wavelength and gain margin are less dependent on the coupling strength and cavity length. The cotresponding threshold current density of the DTWG-DFB lasers is also shown in Fig. 5. As we expected, the threshold current density is reduced with the increase of DTWG's area due to the increase of cavity gain [i.e, modal gain is directly proportional to Lateral confinement factor, see (7)]. For comparison, a discrete $\lambda / 4 \mathrm{DFB}$ laser with $\kappa L=2.0$, the corresponding threshold current density is equal to 2130 and $1970 \mathrm{~A} / \mathrm{cm}^{2}$ for uniform stripe widths of 1.0 and $4.0 \mu \mathrm{m}$, respectively.

Fig. 6 shows the influence of (a) $W_{\text {et }}$ (with $L_{e 1}=140$ $\mu \mathrm{m}$ and $\left.L_{p}=100 \mu \mathrm{m}\right)$, (b) $L_{e 1}$ (with $W_{e t}=3 \mu \mathrm{m}$ and $L_{p}=100 \mu \mathrm{m}$ ), and (c) $L_{p}$ (with $W_{e 1}=3 \mu \mathrm{m}$ and $L_{e 1}=$ $140 \mu \mathrm{m}$ ) on threshold spectra of DTWG-DFB lasets with $\kappa_{0}$ 

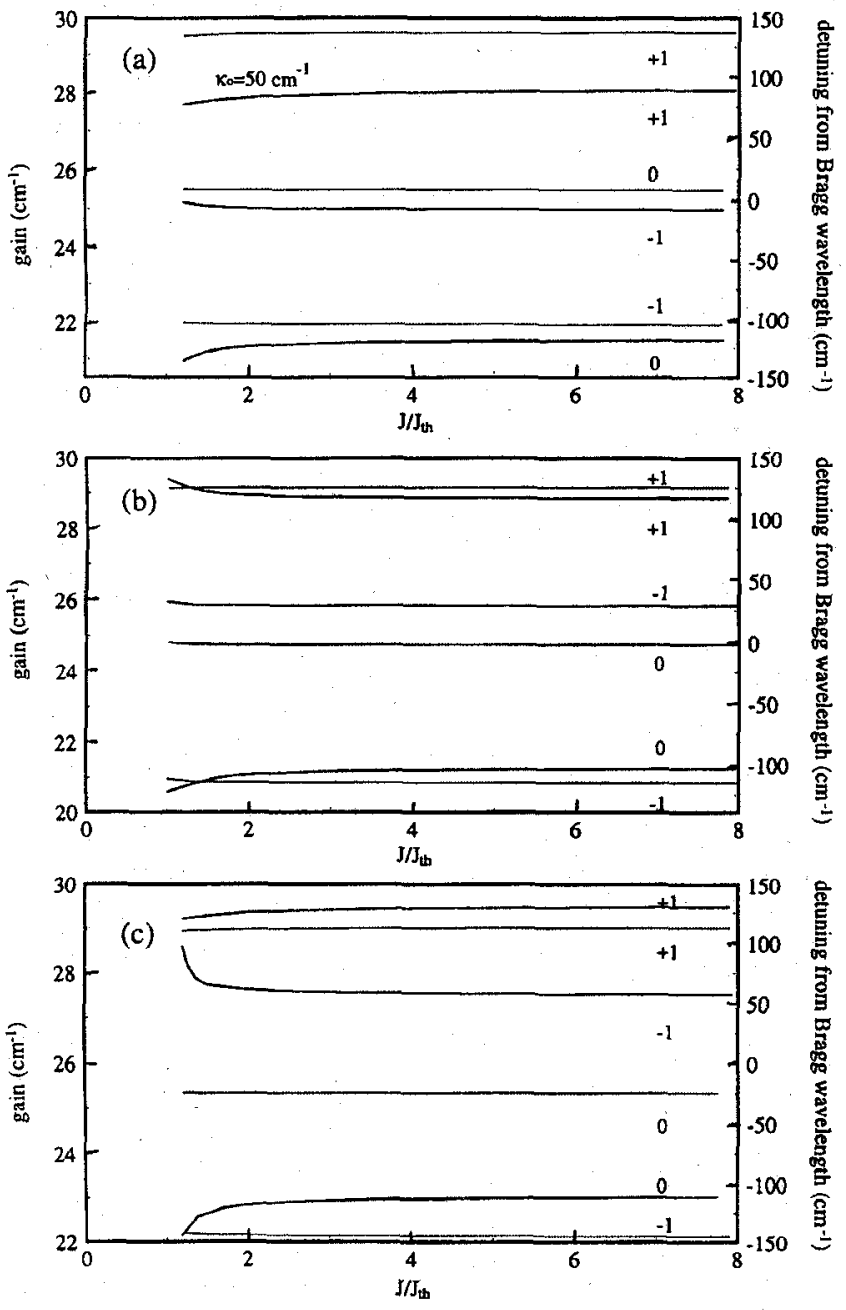

Fig. 7. The variation of modal gain (solid line) and detuning from Bragg wavelength (dotted line) with normalized injection current density of waveguide dimension (a) $W_{e 1}=2.8 \mu \mathrm{m}$, (b) $L_{e 1}=130 \mu \mathrm{m}$, and (c) $L_{p}=86$ $\mu \mathrm{m}$. The laser has $\kappa_{0}=50 \mathrm{~cm}^{-1}$.

equal to $50 \mathrm{~cm}^{-1}$. The width $W_{e 2}$ and length $L_{e 2}$ of the second tapered section is assumed to be varied with $W_{e 1}$ and $L_{e 1}$, respectively. In this figure, the mode " $0, "$ " +1 ," and " -1, , correspond to the dominant mode (band gap mode), the first side mode on the positive detuning side, and the first side mode on the negative detuning side of the band edge. As we can see, band gap mode which is excited by the tapered waveguide is detuned from the Bragg wavelength. In extreme cases, the band edge modes are slightly excited due to the variation of round-trip conditions. Similar properties of emission spectra are observed for devices with $\kappa_{o}=30$ and $80 \mathrm{~cm}^{-1}$. As we can see, the advantages of tapered DFB lasers are that the band gap mode can be oscillated in a wide range of waveguide dimension and with good suppression of side modes. Therefore, the yield rate for single-mode operation is as high as the discrete $\lambda / 4$ or PAR DFB lasers at threshold.

At high injection current, the advent of LSHB as well as reduction of gain margin are expected due to the nonuniform distribution of optical intensity. Furthermore, the stability of single-mode operation is also affected by the nonuniform longitudinal distribution of refractive index. Therefore, in the
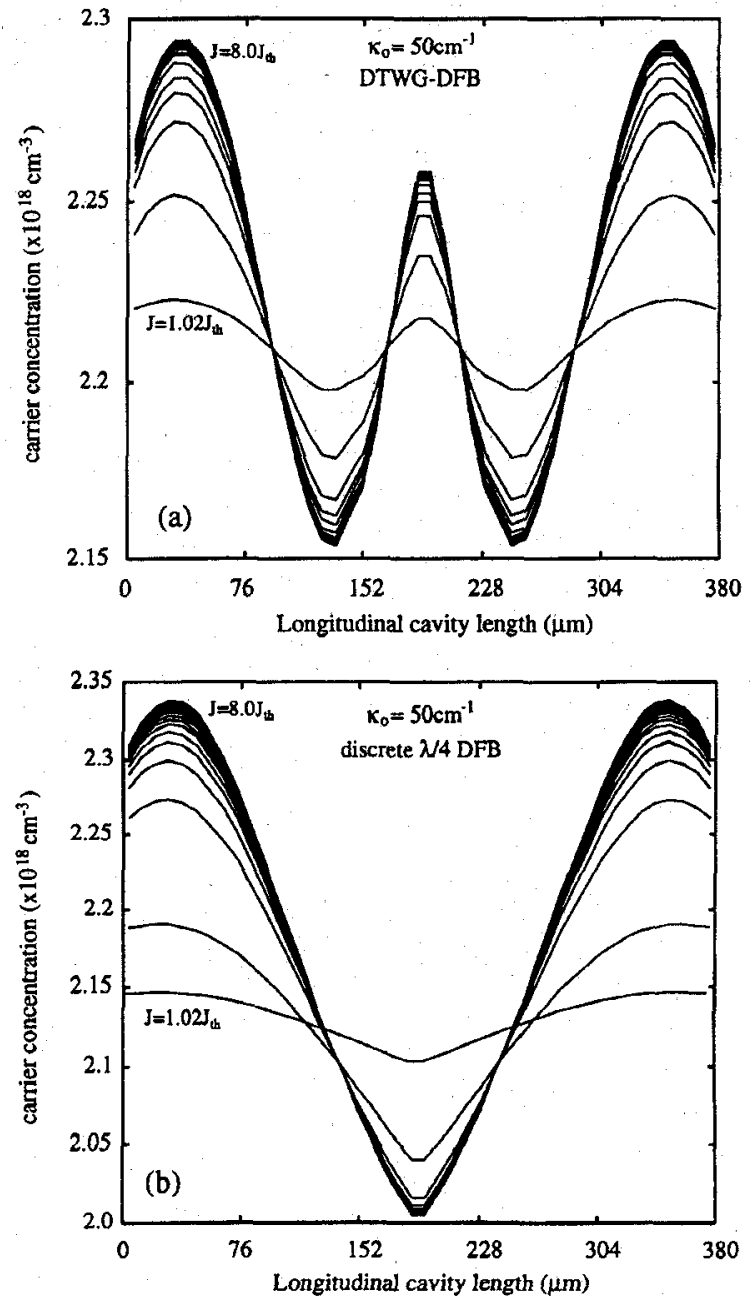

Fig. 8. The variation longitudinal carrier distribution with normalized injection current density of (a) a DTWG-DFB laser and (b) a discrete $\lambda / 4$ DFB laser.

design of a DTWG-DFB laser, the waveguide dimension should be selected with maximum threshold gain margin in order to maintain single-mode operation at high injection current. In the following, we studied the above threshold behavior of DTWG-DFB lasers with waveguide dimension near the position of maximum threshold gain margin.

Fig. 7 examines the above threshold behavior of a DTWGDFB laser with $\kappa_{o}$ equal to $50 \mathrm{~cm}^{-1}$ and the selected waveguide dimension is (a) $W_{e 1}=2.8 \mu \mathrm{m}$ (with $L_{e 1}=140 \mu \mathrm{m}$ and $L_{p}=100 \mu \mathrm{m}$ ), (b) $L_{e 1}=130 \mu \mathrm{m}$ (with $W_{e 1}=3 \mu \mathrm{m}$ and $L_{p}=100 \mu \mathrm{m}$ ), and (c) $L_{p}=86 \mu \mathrm{m}$ (with $W_{e 1}=$ $3 \mu \mathrm{m}$ and $L_{e 1}=140 \mu \mathrm{m}$ ). The width $W_{e 2}$ and length $L_{e 2}$ of the second tapered section is assumed to be equal to $W_{e 1}$ and $L_{e 1}$, respectively. In Fig. 7, the modal gain (solid line) and the detuning wavelength (dotted line) of the oscillation modes (one band gap mode and two band edge modes) are plotted against the normalized injection current density, $J / J_{\mathrm{th}}$. As we can see, the modal gain of the " 0 " mode (dominant mode) slightly increases with the injection current and the " -1 " mode reduces with the injection current such that the gain margin is deteriorated. However, the variation is small, the gain margin between the " 0 " and " -1 " mode is preserved 

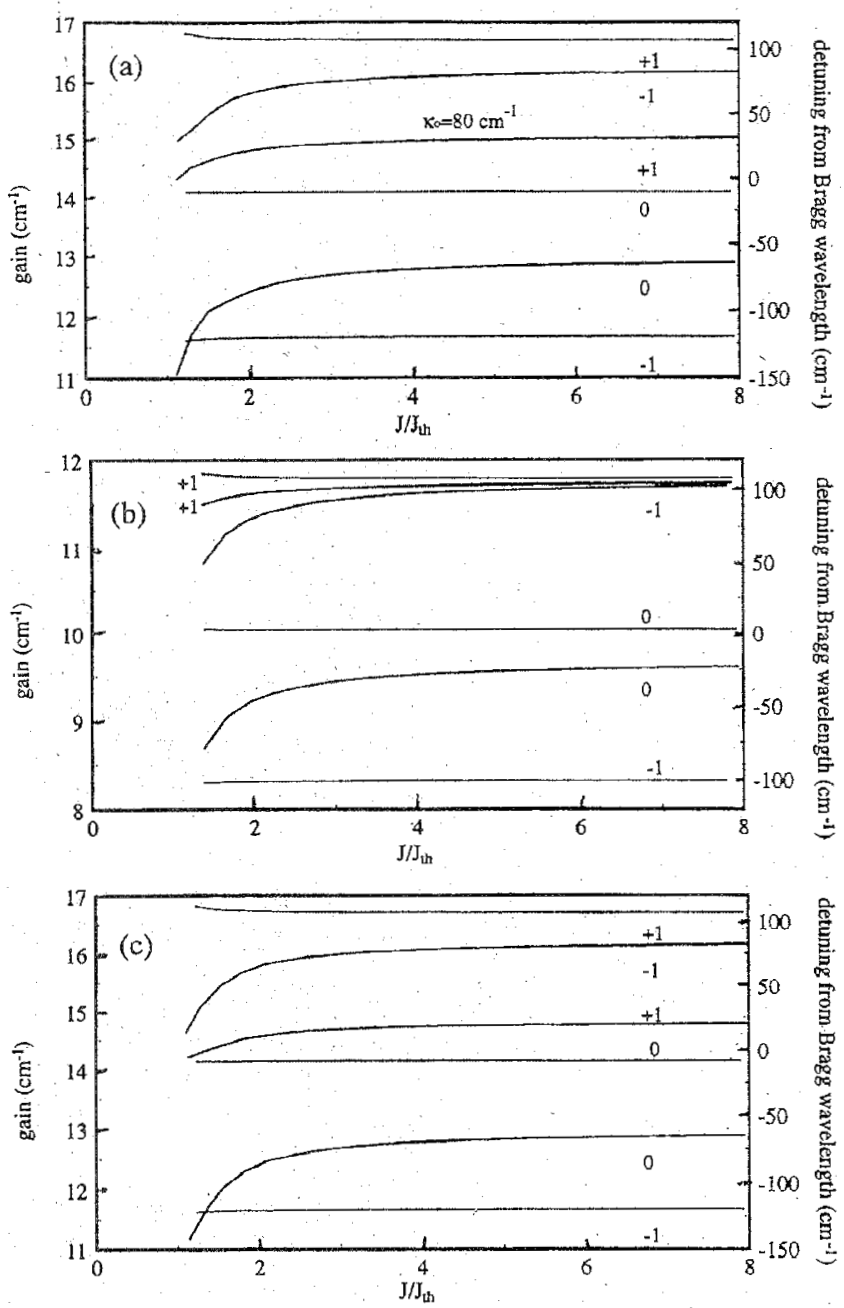

Fig. 9. The variation of modal gain (solid line) and detuning from Bragg wavelength (dotted line) with normalized injection current density of waveguide dimension (a) $W_{e 1}=3.0 \mu \mathrm{m}$, (b) $L_{e 1}=155 \mu \mathrm{m}$, and (c) $L_{p}=105$ $\mu \mathrm{m}$. The laser has $\kappa_{0}=80 \mathrm{~cm}^{-1}$.

at a reasonable large magnitude and single-mode operation is maintained. Fig. 8 compares the LSHB of DTWG [obtained from Fig. 7(a)] and discrete $\lambda / 4$ (same $\kappa_{0} L$ as Fig. 7(a) except uniform stripe width of $2 \mu \mathrm{m}$ wide is assumed) DFB lasers. As we can see, LSHB of carrier concentration is reduced significantly by the DTWG structure.

Fig. 9 shows the variation of modal gain and the detuning wavelength of device with $\kappa_{0}=80 \mathrm{~cm}^{-1}$ against the normalized injection current density. The selected waveguide dimension is (a) $W_{e i}=3.0 \mu \mathrm{m}$ (with ' $L_{e 1}=140 \mu \mathrm{m}$ and $L_{p}=100 \mu \mathrm{m}$ ), (b) $L_{e 1}=155 \mu \mathrm{m}$ (with $W_{e 1}=3 \mu \mathrm{m}$ and $L_{p}=100 \mu \mathrm{m}$ ), and (c) $L_{p}=105 \mu \mathrm{m}$ (with $W_{e 1}=3 \mu \mathrm{m}$ and $L_{e 1}=140 \mu \mathrm{m}$ ). The width $W_{e 2}$ and length $L_{e 2}$ of the second tapered section is assumed to be equal to $W_{e 1}$ and $L_{e 1}$, respectively. It is observed that the modal gain of oscillation modes increases with the injection current such that the gain margin is preserved unchanged. Fig. 10 compares the LSHB of DTWG [obtained from Fig. 9(a)] and discrete $\lambda / 4$ (same $\kappa_{0} L$ as Fig. 9(a) except a uniform stripe width of $2 \mu \mathrm{m}$ wide is assumed) DFB lasers. The results are similar to Fig. 8 as the LSHB of carrier concentration is significantly reduced by
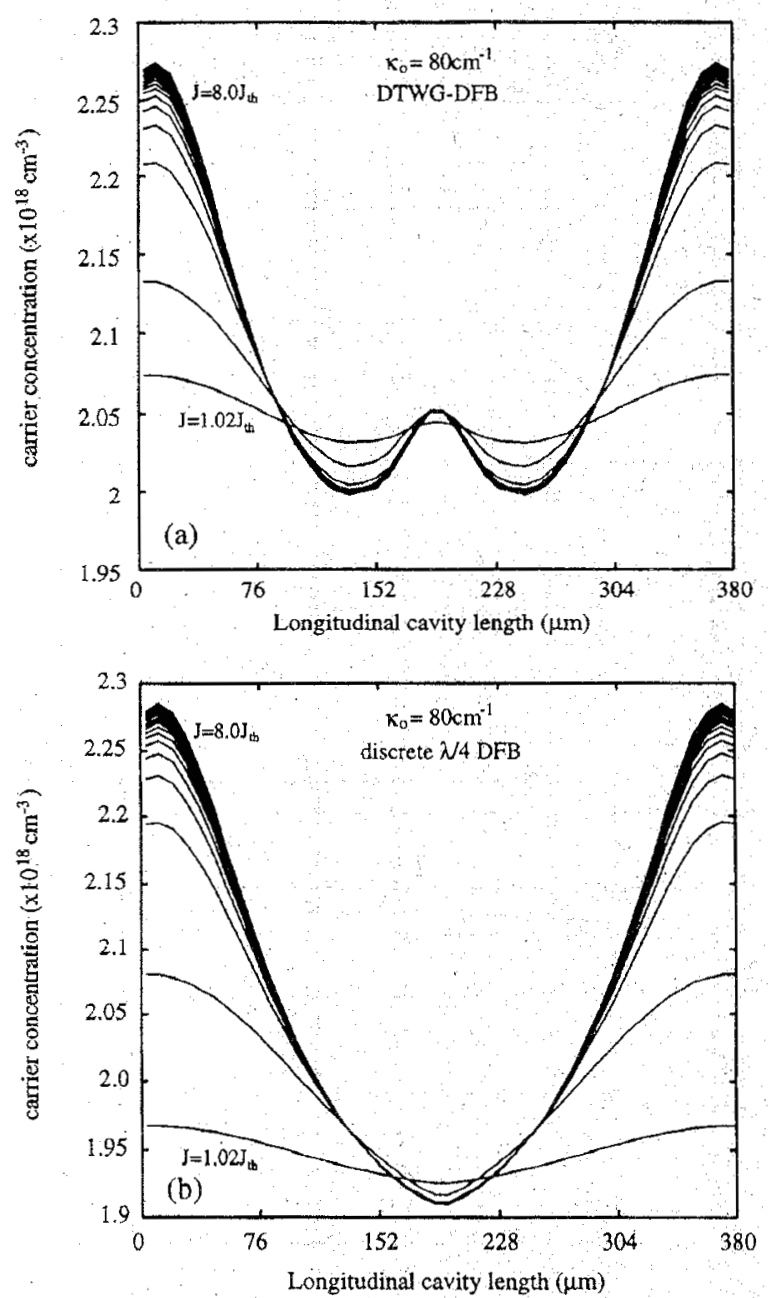

Fig. 10. The yariation longitudinal carrier distribution with nomalized in jection current density of (a) a DTWG-DFB laser and (b) a discrete $\lambda / 4 \mathrm{DFB}$ laser.

the DTWG structure. Hence, it is shown that for a device with large $\kappa_{0}\left(\geq 50 \mathrm{~cm}^{-1}\right)$, gain margin is maintained at high injection current due to the minimization of LSHB by the DTWG structure.

Furthermore, the variation of modal gain and detuning wavelength of a device with $\kappa_{0}=30 \mathrm{~cm}^{-1}$ is shown in Fig. 11. The selected waveguide dimension is (a) $W_{e 1}=$ $2.6 \mu \mathrm{m}$ (with $L_{e 1}=140 \mu \mathrm{m}$ and $L_{p}=100 \mu \mathrm{m}$ ), (b) $L_{e 1}=$ $100 \mu \mathrm{m}\left(W_{e 1}=3 \mu \mathrm{m}\right.$ and $\left.L_{p}=100 \mu \mathrm{m}\right)$, and (c) $L_{p}=$ $70 \mu \mathrm{m}$ (with $W_{e 1}=3 \mu \mathrm{m}$ and $L_{e 1}=140 \mu \mathrm{m}$ ). The width $W_{e 2}$ and length $\dot{L}_{e 2}$ of the second tapered section is assumed to be equal to $W_{e 1}$ and $L_{e 1}$, respectively. The situation is different to devices with $\kappa_{0} \geq 50 \mathrm{~cm}^{-1}$ as the modal gain of " 0 " modes reduces with injection current. In most cases, gain margin can be preserved under high current injection, however, mode hopping is observed between the " 0 " and "-1" mode [see Fig. 11(b)]. Fig. 12 compares the LSHB of DTWG [obtained from Fig. 11(a)] and discrete $\lambda / 4$ (same $\kappa_{0} L$ as Fig. 11(a) except a uniform stripe width of $2 \mu \mathrm{m}$ wide is assumed) DFB lasers. As we can see, LSHB is less significant in discrete $\lambda / 4$ DFB lasers such that the DTWG structure causes an overshoot of carrier concentration at the center of 

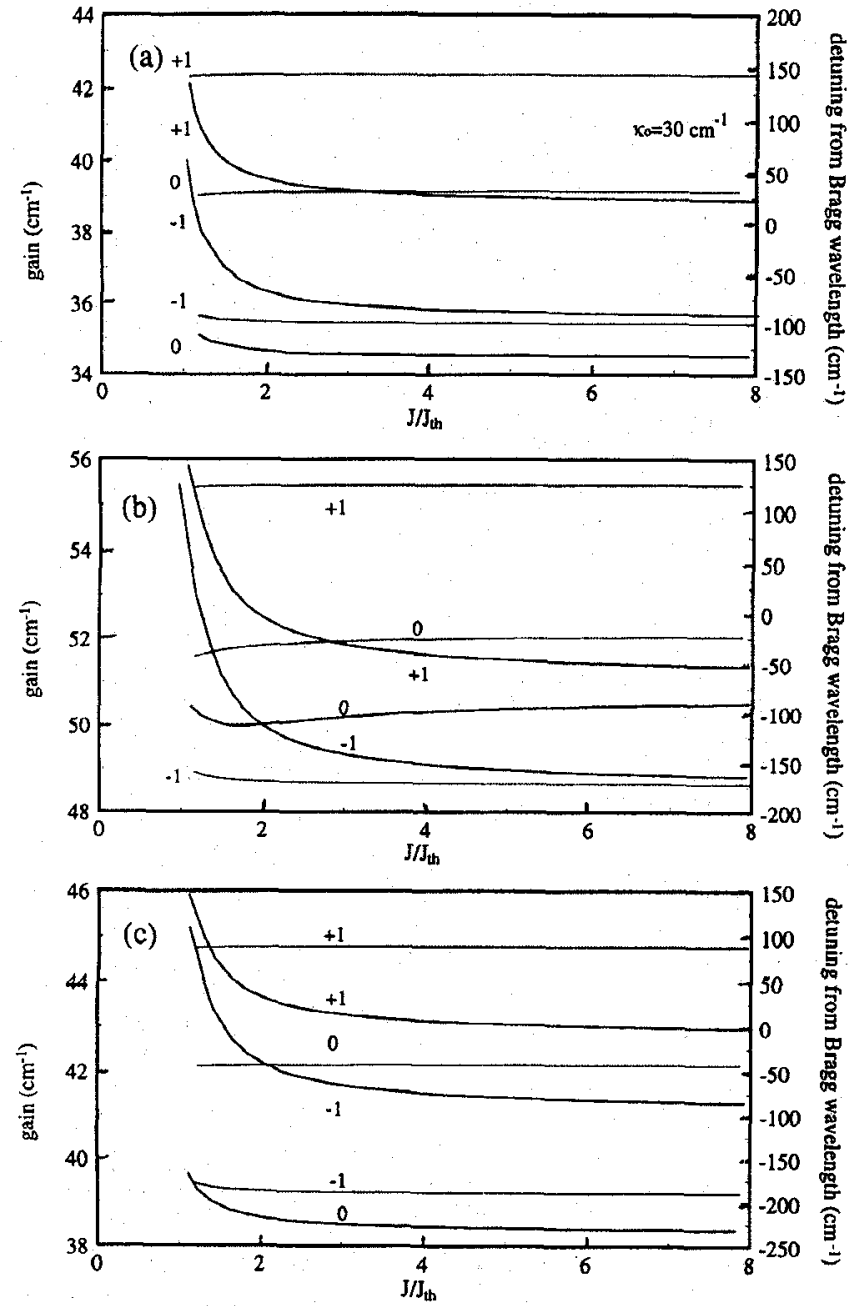

Fig. 11. The variation of modal gain (solid line) and detuning from Bragg wavelength (dotted line) with normalized injection current density of waveguide dimension (a) $W_{e 1}=2.6 \mu \mathrm{m}$, (b) $L_{e 1}=100 \mu \mathrm{m}$, and (c) $L_{p}=70$ $\mu \mathrm{m}$. The laser has $\kappa_{o}=30 \mathrm{~cm}^{-1}$.

the laser [see Fig. 12(a)]. Therefore, in small $\kappa_{o}$ DTWG-DFB lasers, mode hopping may occur due to the change of LSHB (i.e., from concave downward to concave upward). In Figs. 7, 9 , and 11, the output power of the dominant mode at $J=8 J_{t h}$ is equal to or greater than $85 \mathrm{~mW}$.

It is observed that the modal gain as well as the tendency of oscillation modes are affected by $\kappa_{o}$ and waveguide dimension above threshold. As we can see, 1) the magnitude of modal gain is low for lasers with large $\kappa_{0}\left(\geq 50 \mathrm{~cm}^{-1}\right)$, and 2) the modal gain of the " 0 " mode increases (decreases) with injection current for devices with $\kappa_{o} \geq 50 \mathrm{~cm}^{-1}\left(\kappa_{o} \leq 30\right.$ $\mathrm{cm}^{-1}$ ) (similar behavior of " 0 " mode is also observed in PAR DFB lasers with uniform waveguide [1]). This is because for DFB lasers with large coupling strength, the intensity distribution of the optical field is maximum near the center, but for lasers with low coupling strength, the intensity distribution of the optical field is maximum near the facets [1].

Fig. 13 shows the variation of both the effective area, $A_{\text {eff }}$ $\left(\mu \mathrm{m}^{2}\right)$, and power density against the stripe width (i.e., $W_{e 1}$ or $W_{e 2}$ ). In the calculation, it is assumed that $A_{\text {eff }}$ is obtained by multiplying the FWHM optical intensity distribution of the
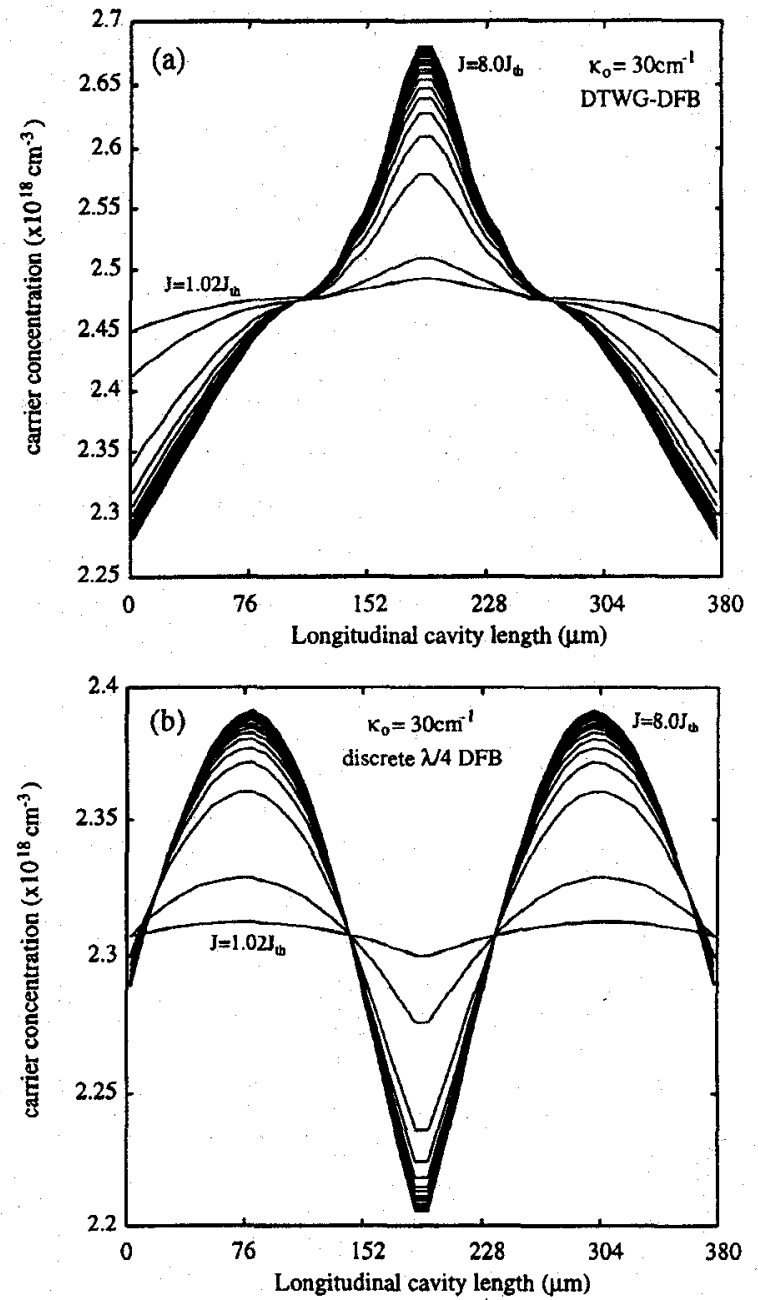

Fig. 12. The variation longitudinal carrier distribution with normalized injection current density of (a) a DTWG-DFB laser and (b) a discrete $\lambda / 4$ DFB laser.

fundamental transverse and lateral modes. As shown in the diagram, a minimum $A_{\text {eff }}$ is located at stripe width equal to $1.5 \mu \mathrm{m}$. The increase in $A_{\text {eff }}$ with the reduction of stripe width (from $1.5 \mu \mathrm{m}$ ) is due to the divergence of lateral field outside the core region. The corresponding power density at the facets is also shown in the figure with the assumption that the output power of device is equal to $10 \mathrm{~mW}$. The power density is roughly inversely proportional to the stripe width, but doubling the width of the stripe (i.e., 2 to $4 \mu \mathrm{m}$ ) only reduces the power density by $30 \%$. Nevertheless, lasers with wide stripe width can reduce power density at facets. It is estimated that the COD level of this material system is greater than $20 \mathrm{MW} / \mathrm{cm}^{2}$ (the result is based on the experimental results given in [8] as their devices with similar material composition exhibit $\mathrm{CW}$ output peak power of $430 \mathrm{~mW}$ and $800 \mathrm{~mW}$ pulsed mode). Therefore, the maximum $\mathrm{CW}$ output power of our proposed device can be greater than $350 \mathrm{~mW}$ with low-threshold current density.

\section{DTWG DFB Lasers with AR-Cleaved Facets}

In DFB lasers, external optical feedback has significant influence on its spectrum profile as well as longitudinal photon distribution [16]. In the design of $\lambda / 4$ phase-shifted DFB 


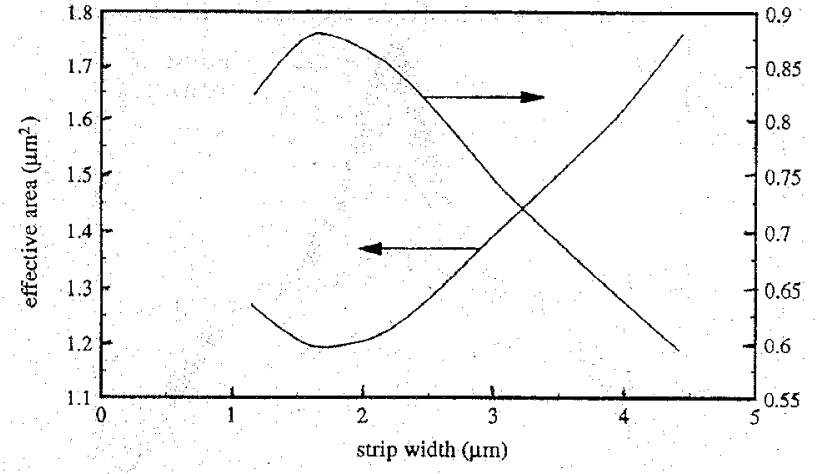

Fig. 13. The variation of effective area $\left(A_{\text {eff }}\right)$ and power density with the stripe width.

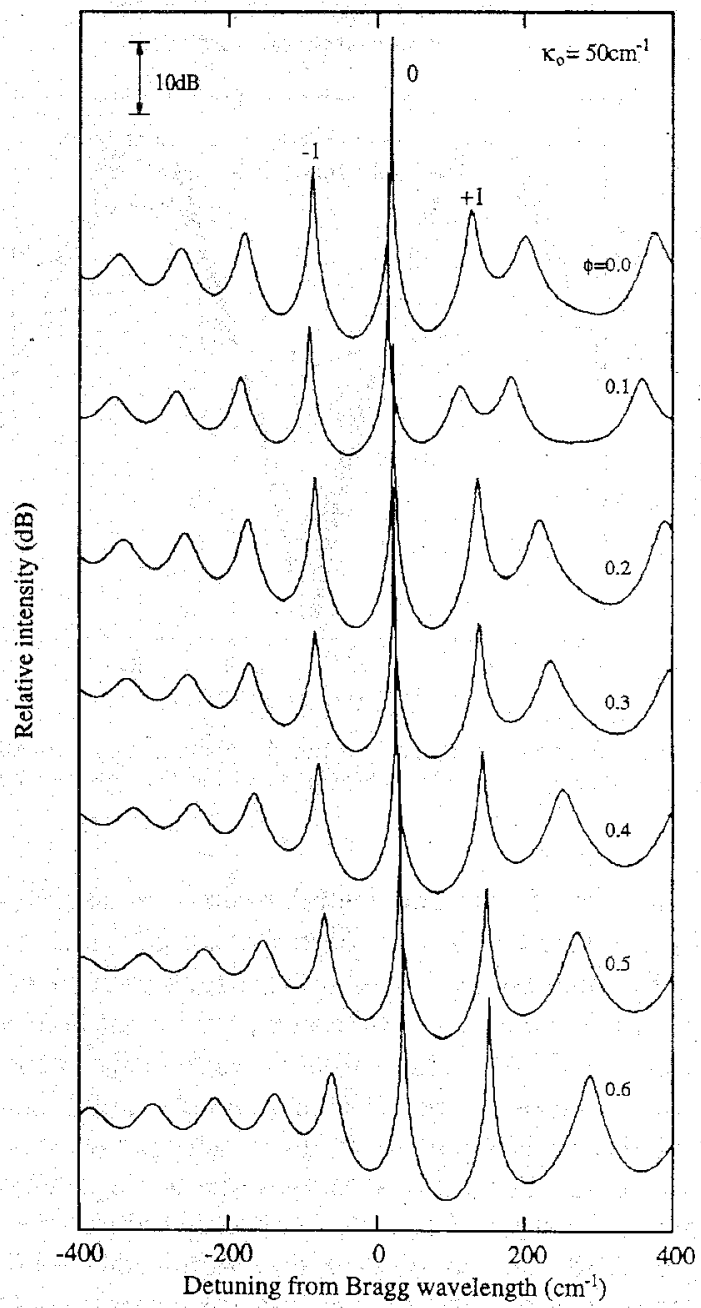

Fig. 14. The threshold spectrum profile of the DTWG-DFB laser with AR-HR coatings.

lasers, reflection of facets is avoided due to low yield rate of side-mode operation. Nevertheless, uniform grating DFB lasers with AR-HR coatings are usually employed to enhance the output efficiency of the devices. It is expected that the magnitude of facet's reflection and phase in DTWG-DFB lasers have significant influence on: 1) its spectrum profile; 2) its distribution of photon density; and 3) its power ratio between AR- and HR-facets. This is because of the roundtrip phase requirement that is the sum of the phase of: 1) the

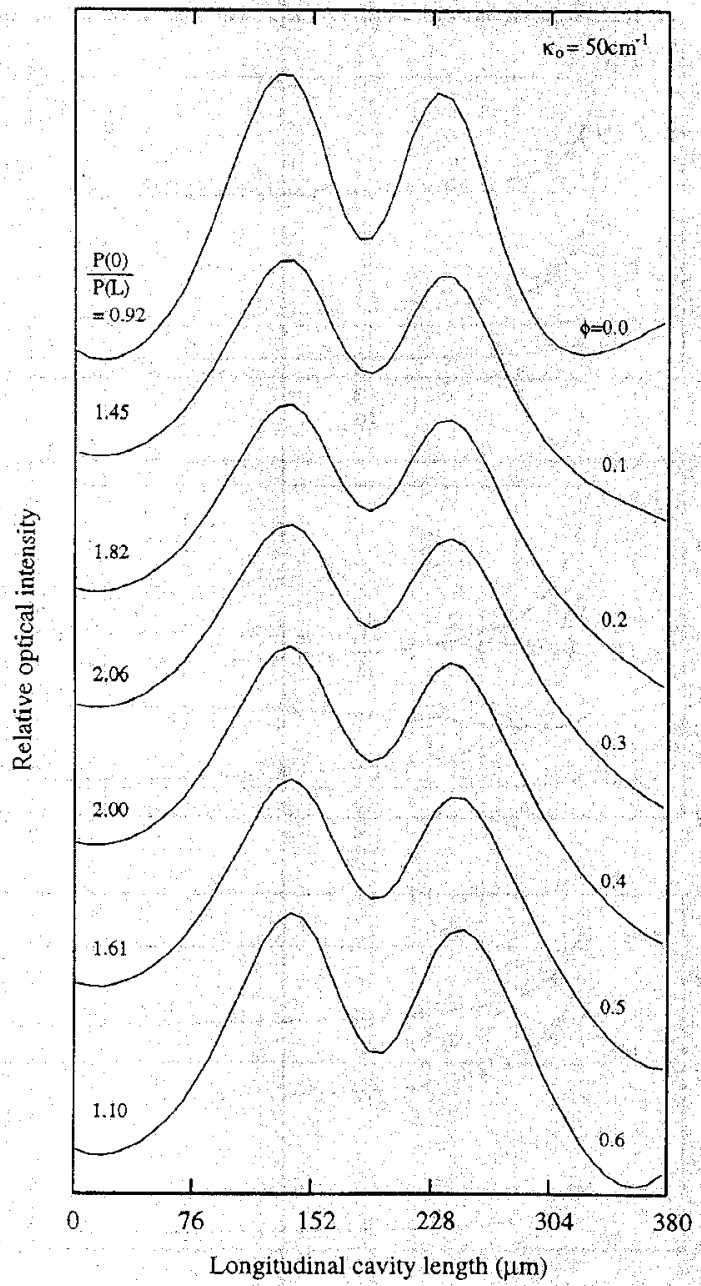

Fig. 15. The threshold photon-density distribution of the DTWG-DFB laser with AR-cleaved facets.

tapered section with AR coating; and 2) the tapered section with HR coating. The PAR should be equal to $2 \pi$.

Therefore, the influence of facet's phase on the tapered section with HR coating is analyzed. It is assumed that the device with $\kappa_{0}=50 \mathrm{~cm}^{-1}, W_{e 1}=W_{e 2}=3 \mu \mathrm{m}, W_{p}=$ $1 \mu \mathrm{m}, L_{e 1}=L_{e 2}=135 \mu \mathrm{m}$, and $L_{p}-110 \mu \mathrm{m}$. The left and right facet reflectivity $\left(r_{L}\right.$ and $r_{R}$, respectively) is equal to $0.0 \angle 0^{\circ}$ and $0.55 \angle 2 \pi \phi^{\circ}$, respectively. Fig. 14 shows the threshold spectrum profile of the device for $\phi$ varies between 0 and 0.6. As shown in the figure, the " 0 " mode (band-gap mode) is dominated at this range of $\phi$, but the " -1 " and " +1 " modes are excited for the range of $\phi$ varies between 0.6 and 1.0. The longitudinal photon-density distribution and the corresponding power ratio (i.e, $P(0) / P(L)$ ) are shown in Fig. 15. The requirement of single-mode and power tatio (i.e., $P(0) / P(L)>1$ ) can be obtained in a large range of $\phi$ (i.e., $0.1 \geq \phi \geq 0.6$ ) and the yield rate can be as high as $50 \%$. We also examine the case $r_{R}=0.922 \pi \phi^{\circ}$ of the DTWG-DFB laser. It is found that the yield rate for singlemode operation with $P(0) / P(L)>1$ is less than $40 \%$ due to the fact that the high facet's reflection excites band-edge modes. Therefore, DTWG-DFB lasers with AR-cleaved facets seem to be a better choice. 
In the above analysis, the effective refractive index $n_{\text {eff }}$ is calculated based on the assumption that only fundamental lateral modes are excited in the above waveguide dimension of DTWG-DFB lasers. However, we have to check whether high-order lateral mode is excited in the above waveguide dimension. In fact, it can be shown by using the laser model in [11] that high-order lateral mode is suppressed by the waveguide design. Therefore, our assumption used in the above calculation is satisfied and the influence of first-order lateral mode can be ignored.

\section{CONCLUSION}

The steady-state behavior of DTWG-DFB lasers is investigated theoretically. We have shown that the threshold gain margin and spectrum profile are dependent on the waveguide dimension. However, in a wide range of waveguide dimension, band-gap mode can be excited with sufficient threshold gain margin. On the other hand, the influence of LSHB has negligible influence on the single-mode operation, especially for devices with large coupling strength (i.e., $\kappa_{o} \geq 50 \mathrm{~cm}^{-1}$ ) and this is desirable for DFB lasers for the reasons mentioned in Section I. High-power performance of DTWG-DFB lasers can also be enhanced by using AR-cleaved facets with yield rate (i.e., single-mode and power ratio $>1$ ) of $50 \%$. Compared with the existing design of DFB lasers, a DTWG with BH has much better performance for the following reasons.

1) Controllability of lateral electrical and optical confinement.

2) High SMSR is maintained at high injection current for devices with large coupling strength.

3) Without changing the grating period, the lasing mode can be tuned from the Bragg wavelength by modifying the waveguide dimension.

4) It is a simple processing technique and compatible with the existing fabrication technologies of the semiconductor lasers.

5) Power density is reduced at facets by the wide-stripewidth waveguide design such that the chance of mirror damage due to thermal effects can be minimized.

\section{APPENDIX A}

We assumed the field profiles in the transverse $(x)$ and lateral $(y)$ directions satisfy

$$
\begin{aligned}
& {\left[\frac{\partial^{2}}{\partial x^{2}}+n_{o}^{2} k_{o}^{2}-\beta_{\mathrm{eff}}^{2}\right] \phi_{o}=0} \\
& {\left[\frac{\partial^{2}}{\partial y^{2}}+\left(\beta_{\mathrm{eff}}^{2}-\beta^{2}\right)\right] \psi_{o}=0}
\end{aligned}
$$

where $n_{o}$ is the refractive index profile of the waveguide, $\beta_{\text {eff }}$ is the effective propagation coefficient in lateral direction, and $\beta$ is a propagation constant to be determined. $\phi_{o}$ and $\psi_{o}$ are the fundamental transverse and lateral field distribution, respectively. The effective refractive index $n_{\text {eff }}$ given in (4) is defined as [12]

$$
n_{\mathrm{eff}}=\beta_{\mathrm{eff}} / k_{0}
$$

where $k_{o}=2 \pi / \lambda_{o}$. The transverse confinement factor $\Gamma_{x}$ is expressed as

$$
\Gamma_{x}=\int_{\substack{\text { active } \\ \text { layer }}} \phi_{0}^{2} d x
$$

and the lateral confinement factor $\Gamma_{y}$ is given by

$$
\Gamma_{y}=\int_{0}^{w} \psi_{o}^{2} d y
$$

where $w$ is the width of the waveguide. The longitudinal coupling coefficient $\kappa$ is given by

$$
\kappa=\frac{1}{2 \beta_{o}} \int_{0}^{w} \int_{\substack{\text { grating } \\ \text { layer }}} k_{o}^{2} A_{-2} \phi_{o}^{2} \psi_{o}^{2} d x d y \approx \kappa_{o} \Gamma_{y}
$$

where $A_{-2}$ is the Fourier coefficient of the dielectric grating, $\beta_{o}$ is the propagation constant at Bragg frequency, and $\kappa_{o}$ is the coupling coefficient of PAR.

\section{APPENDIX B}

A general method to handle nonuniform distributions of structure and material parameters such as confinement factor and effective refractive index is to split the laser into cascaded sections [15]. In each section, it is assumed that the laser parameters are assumed to be constant. If the DFB laser has cavity length $L$ and consisting of a number of sections $(n=1, \ldots, M)$, the length of the $n$th section is equal to $\Delta z_{n}=L / M$. The optical fields within the $n$th section can be described by the transfer matrix $T_{n}$ as (B1), shown at the bottom of the page, where $g_{n}=\frac{1}{2} \Gamma_{n}\left(\Gamma_{x} G-\alpha_{s}\right)$ and $\gamma_{n}^{2}=\left(g_{n}+i \delta \beta_{n}\right)^{2}+\kappa_{n}^{2}$. The longitudinal variation of net modal gain $g_{n}$ and the coupling coefficient $\kappa_{n}$ are constant in each section but varied with the section number. Therefore, the coupled wave equations (1) can be expressed as

$$
\begin{aligned}
{\left[\begin{array}{l}
F \\
R
\end{array}\right]_{n=M+1} } & =\left[\prod_{m=1}^{N} T_{m}\right]\left[\begin{array}{l}
F \\
R
\end{array}\right]_{n=1} \\
& =\left[\begin{array}{ll}
T T_{11} & T T_{12} \\
T T_{21} & T T_{22}
\end{array}\right]\left[\begin{array}{l}
F \\
R
\end{array}\right]_{n=1} .
\end{aligned}
$$

The lasing conditions for the longitudinal modes can be evaluated from the boundary conditions at the laser facets

$$
F_{1}=r_{L} R_{1} \quad \text { and } \quad R_{M+1}=r_{R} F_{M+1}
$$

$$
T_{n}=\left[\begin{array}{cc}
\cosh \left(\gamma_{n} \Delta z_{n}\right)+\frac{g_{n}+i \delta \beta_{n}}{\gamma_{n}} \sinh \left(\gamma_{n} \Delta z_{n}\right) & \frac{i \kappa_{n}}{\gamma_{n}} \sinh \left(\gamma_{n} \Delta z_{n}\right) \\
\frac{-i \kappa_{n}}{\gamma_{n}} \sinh \left(\gamma_{n} \Delta z_{n}\right) & \cosh \left(\gamma_{n} \Delta z_{n}\right)-\frac{g_{n}+i \delta \beta_{n}}{\gamma_{n}} \sinh \left(\gamma_{n} \Delta z_{n}\right)
\end{array}\right]
$$


where the reflectivities at the left and tight facets of the laser are assumed to be $r_{L}$ and $r_{R}$. By substituting (B3) into (B2), we get the determinant $D$ of $(\mathrm{B} 2)$ as

$$
D=T T_{22}-r_{R} T T_{12}+r_{L} T T_{21}-r_{R} r_{L} T T_{11}
$$

Equating the real part and imaginary part of $D$ to zero, the threshold gain and lasing frequency of DTWG-DFB can be calculated.

\section{REFERENCES}

[1] H. Soda, Y. Kotaki, H. Sudo, H. Ishikawa, S. Yamakoshi, and H. Imai, "Stability in single longitudinal mode operation in GaInAsP/InP phaseadjusted DFB lasers," IEEE J. Quantum Electron, vol. QE-23, pp. $804-814,1987$.

[2] J. E. A. Whiteaway, G. H. B. Thompson, A. J. Collar, and C. J. Armistead "The design and assessment of $\lambda / 4$ phase-shifted DFB laser structure," IEEE J. Quantum Electron., vol. 25, pp. 1261-1279, 1989.

[3] J. Kinoshita, K. Ohtsuka, H. Agatsuma, A. Tanaka, T. Matsuyama, A. Makuta, and $H$. Kobayashi, Performance of $1.5 \mu \mathrm{m}$ DFB lasers with a narrow stripe region," IEEE J. Quantum Electron., vol: 27, pp. $1759-1765,1991$.

[4] P. Zhou and G. S. Lee, "Chirped grating $\lambda / 4$ shifted DFB laser with uniform longitudinal field distribution," Electron. Letr., vol. 26, pp. $1660-1661,1990$

[5] H. Hillmer, K. Magari, and Y. Suzuki, "Chirped gratings for DFB laser diodes using bent waveguides," IEEE Photori. Technol. Lett., vol. 5, pp. 10-12, 1993.

[6] J. S. Yoo, H. H. Lee, and P. Zory, "Temperature rise at mirror facet of CW semiconductor lasers;" IEEE J Quantum Electron, vol. 28, pp$635-639,1992$.

[7] C. H. Henry, "Performance of distributed feedback lasers designed to favor the energy gap mode;" IEEE J. Quantum Electron., vol. QE-21, pp. $1913-1918,1985$

[8] H. Asonen, A. Ovtchinnikov, G. Zhang, J. Nappi, P. Savolainen, and M. Pessa, "Aluminum-free $980 \mathrm{~nm}$ pump lasers," IEEE J. Quantum Electron., vol 30 pp $415-423,1994$

[9] Y. K. Chen, M. C. Wu, J. M. Kuo, M. A. Chin, and A. M. Sergent "Self-aligned InGaAs/GaAs/InGaP quantum well lasers prepared by gassource molecular beam epitaxy with two growth steps," Appl. Phys. Lett. vol. 59, no. 23 , pp. $2929-2931,1991$
[10] D. Z, Grabuzov, N. J. Antonishkis, S. N Zhigulin, N. D. L1' inskaya, A. V. Kochergin, D. A. Lifshitz, E U. Rafilov, and M, V Fuksman, "High-power buried InGaAsP/GaAs $(\lambda=0.8 \mu \mathrm{m})$ laser diodes." Appl. Phys. Lett, vol, 62, no. 10, pp. 1062-1064, 1993

[11] S. F. Yu, "A quasi-three-dimensional large-signal dynamie model of distributed feedback lasers;" IEEE J Quantum Electron., yol. 32, pp. $424-432,1996$.

[12] M. J. Adams, An Introduction to Optical Waveguide. New York Wiley; 1981; ch. 6.

[13] M. Ohkubo, T. Ijichi, A. Iketani, and T. Kikuta, Aluminum free InGaAs/GaAs/InGaAsP/InGaP GRINSCH SL-SQW lasers at $0.98 \mu \mathrm{m}$," Electron. Lett. vol. 28, pp. $1149-1150,1992$

[14] M. Yamada and K. Sakuda, Analysis almost-periodic distributed feedback slab. waveguides via a fundamental matrix approach", Appl. Opt. vol. 26, pp. $3473-3478,1987$

[15] L. M. Zhang and J. E. Carroll, "Large signal dyaamic model of the DFB Iaser," IEEE J Quantum Electron, vol. 28, pp 604-611, 1992

[16] S. F. Yu, L. M. Zhang, R G S Plumb, and I. E Carroll, Effect of external reflectors on radiation profile of grating coupled surface emitting lasers," Proc. Inst Elect. Eng, vol 140. Part J, pp. 30-38, 1993.

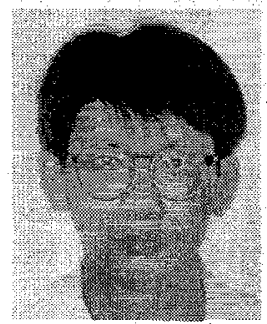

S. F. Yu received the B.Eng degree (with Departmental Prize) in electronic engineering from London University, University College, England, in 1990 and the Ph.D. degree in optoelectronic engineerng from Cambridge University, Røbinson College, England, in 1993.

In 1994, he joined the Department of Electrical \& Electronic Engineering, University of Hong Kong, where he is a Lecturer. His main research topics include wayelength selectivity of grating coupled waveguides, transient properties of semiconductor laser diodes, and the design of optoelectronie integrated circuit. He currently conducts the development of high-performance semiconductor lasers for application in high-speed communication systems. $\mathrm{He}$ is also involved in the investigation of the influence of acoustic wave in semiconductor materials. He has published over 30 technical papers.

Dr. Yn is a Fellow and Honorary Scholar of Cambridge Commonwealth Trust and holds a Croucher Foundation scholarship and an oversea research student award while studying for the Doctoral program. 